\title{
Integrated schedule planning with supply-demand interactions for a new generation of aircrafts
}

Bilge Atasoy, Matteo Salani and Michel Bierlaire

\begin{abstract}
We present an integrated schedule planning model where the decisions of schedule design, fleeting and pricing are made simultaneously. Pricing is integrated through a logit demand model where itinerary choice is modeled by defining the utilities of the alternative itineraries. Spill and recapture effects are appropriately incorporated in the model by using a logit formulation similar to the demand model. Furthermore class segmentation is considered so that the model decides the allocation of the seats to each cabin class. We propose a heuristic algorithm based on Lagrangian relaxation to deal with the high complexity of the resulting mixed integer nonlinear problem.
\end{abstract}

\section{Introduction and Motivation}

Transportation demand is constantly increasing in the last decades for both passenger and freight transportation. According to the statistics provided by the Association of European Airlines (AEA), air travel traffic has grown at an average rate over $5 \%$ per year over the last three decades. Given the trends in the air transportation, actions need to be taken both in supply operations and the demand management to have a demand responsive transportation capacity for the sustainability of transportation.

This study is in the context of a project regarding the design of a new air transportation concept, Clip-Air, at Ecole Polytechnique Fédérale de Lausanne. Clip-Air is designed to introduce flexibility in various aspects including multi-modality, mod-

Bilge Atasoy

Transport and Mobility Laboratory, ENAC, EPFL, e-mail: bilge.kucuk@epfl.ch

Matteo Salani

Dalle Molle Institute for Artificial Intelligence (IDSIA), Lugano, e-mail: matteo.salani@idsia.ch

Michel Bierlaire

Transport and Mobility Laboratory, ENAC,EPFL, e-mail: michel.bierlaire@epfl.ch 
ularity, demand management and robustness. The main characteristic of Clip-Air is that it simplifies the fleet management by allowing to decouple the carrying (wing) and the load (capsule) units. The wing carries the flight crew and the engines, and the capsules carry the passengers/freight. The separation of passengers from the pilot and the fuel has several advantages in terms of security. Furthermore, capsules are modular detachable units such that the transportation capacity can be modified according to the demand. Clip-Air is already tested in a simulation environment and the aircraft design is shown to be feasible. From the operations viewpoint, a preliminary analysis on the potential advantages of Clip-Air is presented in [1]. An integrated schedule design and fleet assignment model is developed to compare the performance of Clip-Air with standard aircraft. In this paper we focus on modeling aspects and solution methods. Therefore we present the model for standard aircraft where we further integrate supply-demand interactions into the scheduling model.

\section{Related Literature}

Supply-demand interactions are studied in the context of fleet assignment with different perspectives. In an itinerary-based setting, [2] consider the spill and recapture effects separately for each class resulting from insufficient capacity. Similarly, [3] study the network effects including the demand adjustment in case of flight cancellations.

Advanced supply and demand interactions can be modeled by letting the model to optimize itinerary's attributes (e.g., the price). [5] integrate discrete choice modeling into the single-leg, multiple-fare-class revenue management model that determines the subset of fare products to offer at each point in time. [4] develops a market-oriented integrated schedule design and fleet assignment model with integrated pricing decisions. Several specifications are proposed for the pricing model including discrete choice models such as logit model and nested logit model where the explanatory variable is taken as the fare price.

\section{Integrated schedule planning model}

We develop an integrated schedule design, fleet assignment and pricing model. The pricing decision is integrated through a logit model. The utility of the itineraries are defined as below:

$$
V_{i}^{h}=\beta_{\text {fare }}^{h} p_{i}^{h}+\beta_{\text {time }}^{h} \text { time }_{i}+\beta_{\text {stops }}^{h} \text { nonstop }_{i},
$$

where $p_{i}^{h}$ is the price of itinerary $i \in I$ for class $h \in H$, time $e_{i}$ is a dummy variable which is 1 if departure time is between 07:00-11:00, and nonstop $p_{i}$ is a dummy variable which is 1 if $i$ is a non-stop itinerary. The coefficients of these variables, which 
are specific to each class $h$, are estimated with maximum likelihood estimation. Total expected demand for each market segment $s \in S\left(D_{s}^{h}\right)$ is attracted by the itineraries in the segment $\left(I_{S} \in I\right)$ according to the logit formula in equation (2).

$$
\tilde{d}_{i}^{h}=D_{s}^{h} \frac{\exp \left(V_{i}^{h}\right)}{\sum_{j \in I_{s}} \exp \left(V_{j}^{h}\right)} \quad \forall s \in S, h \in H, i \in I_{s} .
$$

In case of capacity shortage some passengers, who can not fly on their desired itineraries, may accept to fly on other available itineraries in the same market segment. This effect is referred as spill and recapture effect. In this paper we model accurately the spill and recapture in order to better represent the demand. We assume that the spilled passengers are recaptured by the other itineraries with a recapture ratio based on a logit choice model as given by the equation (3). We include nopurchase options $\left(I_{s} \in I_{S}\right)$, which are the itineraries offered by competitive airlines, in order to represent the lost passengers in a more realistic way.

$$
b_{i, j}^{h}=\frac{\exp \left(V_{j}^{h}\right)}{\sum_{k \in I_{s} \backslash i} \exp \left(V_{k}^{h}\right)} \quad \forall s \in S, h \in H, i \in\left(I_{s} \backslash I_{s}^{\prime}\right), j \in I_{s} .
$$

The integrated model, which considers a single airline, is provided in Figure 1. Let $F$ be the set of flight legs, there are two subsets of flights one being mandatory flights $\left(F_{M}\right)$, which should be flown, and the other being the optional flights $\left(F_{O}\right)$ which can be canceled in the context of schedule design decision. $A$ represents the set of airports and $K$ is for the set of fleet. The schedule is represented by time-space network such that $N(k, a, t)$ is the set of nodes in the time-line network for plane type $k$, airport $a$ and time $t \in T . \operatorname{In}(k, a, t)$ and $\operatorname{Out}(k, a, t)$ are the sets of inbound and outbound flight legs for node $(k, a, t)$.

Objective (4) is to maximize the profit which is calculated with revenue for business and economy demand, that takes into account to lost revenue due to spill, minus operating costs. Operating cost for flight $f$ when using fleet type $k$ is represented by $C_{k, f}$ which is associated with a binary variable of $x_{k, f}$ that is one if a plane of type $k$ is assigned to flight $f$. Constraints (5) ensure the coverage of mandatory flights which must be served according to the schedule development. Constraints (6) are for the optional flights that have the possibility to be canceled. Constraints (7) are for the flow conservation of fleet, where $y_{k, a, t^{-}}$and $y_{k, a, t^{+}}$are the variables representing the number of type $k$ planes at airport $a$ just before and just after time $t$. Constraints (8) limit the usage of fleet by $R_{k}$ for fleet type $k$. It is assumed that the network configuration at the beginning and at the end of the day is the same in terms of the number of planes at each airport, which is ensured by the constraints (9).

The assigned capacity for a flight should satisfy the demand for the corresponding itineraries considering the spill effects as maintained by the constraints (10). Similarly when a flight is canceled, all the related itineraries should not realize any demand. $\delta_{i, f}$ is a binary parameter which is 1 if itinerary $i$ uses flight $f$ and enables us to have itinerary-based demand. We let the allocation of business and economy 


$$
\begin{aligned}
& \operatorname{Max} \sum_{s \in S} \sum_{h \in H} \sum_{i \in\left(I_{s} \backslash I_{s}^{\prime}\right)}\left(d_{i}^{h}-\sum_{\substack{j \in I_{s} \\
i \neq j}} t_{i, j}^{h}+\sum_{\substack{j \in\left(I_{s} \backslash_{s}^{\prime}\right) \\
i \neq j}} t_{j, i}^{h} b_{j, i}^{h}\right) p_{i}^{h}-\sum_{\substack{k \in K \\
f \in F}} C_{k, f} x_{k, f} \\
& \begin{aligned}
\text { s.t. } & \sum_{k \in K} x_{k, f}=1 \\
& \sum x_{k, f} \leq 1
\end{aligned} \\
& \sum_{k \in K} x_{k, f} \leq 1 \\
& y_{k, a, t^{-}}+\sum_{f \in \operatorname{In}(k, a, t)} x_{k, f}=y_{k, a, t^{+}}+\sum_{f \in O u t(k, a, t)} x_{k, f} \\
& \sum_{a \in A} y_{k, a, t_{n}}+\sum_{f \in C T} x_{k, f} \leq R_{k} \\
& \forall f \in F^{M} \quad(5) \\
& \forall f \in F^{O} \\
& \forall[k, a, t] \in N \quad(7 \\
& \forall k \in K \\
& y_{k, a, \min E_{a}^{-}}=y_{k, a, \max E_{a}^{+}} \\
& \sum_{s \in S} \sum_{i \in\left(I_{s} \backslash I_{s}^{\prime}\right)} \delta_{i, f} d_{i}^{h}-\sum_{\substack{j \in I_{s} \\
i \neq j}} \delta_{i, f} t_{i, j}^{h}+\sum_{\substack{j \in\left(I_{I} \backslash I_{s}^{\prime}\right) \\
i \neq j}} \delta_{i, f} t_{j, i}^{h} b_{j, i}^{h} \leq \sum_{k \in K} \pi_{k, f}^{h} \\
& \forall k \in K, a \in A \quad \text { (9) } \\
& \forall h \in H, f \in F(10) \\
& \sum_{h \in H} \pi_{k, f}^{h}=Q_{k} x_{k, f} \\
& \forall f \in F, k \in K(11) \\
& \sum_{\substack{j \in I_{s} \\
i \neq j}} t_{i, j}^{h} \leq d_{i}^{h} \\
& \forall s \in S, h \in H, i \in\left(I_{s} \backslash I_{s}^{\prime}\right)( \\
& \tilde{d}_{i}^{h}=D_{s}^{h} \frac{\exp \left(V_{i}^{h}\right)}{\sum_{j \in I_{s}} \exp \left(V_{j}^{h}\right)} \\
& \forall s \in S, h \in H, i \in I_{s}(13) \\
& b_{i, j}^{h}=\frac{\exp \left(V_{j}^{h}\right)}{\sum_{k \in I_{s} \backslash i} \exp \left(V_{k}^{h}\right)} \\
& \forall s \in S, h \in H, i \in\left(I_{s} \backslash I_{s}^{\prime}\right), j \in I_{s}(14) \\
& x_{k, f} \in\{0,1\} \\
& \forall k \in K, f \in F(15) \\
& y_{k, a, t} \geq 0 \\
& \forall[k, a, t] \in N(16) \\
& \pi_{k, f}^{h} \geq 0 \\
& \forall h \in H, k \in K, f \in F \text { (17) } \\
& d_{i}^{h} \leq \tilde{d}_{i}^{h} \\
& \forall h \in H, i \in I \text { (18) } \\
& 0 \leq p_{i}^{h} \leq U B_{i}^{h} \\
& \forall h \in H, i \in I \text { (19) } \\
& t_{i, j}^{h}, b_{i, j}^{h} \geq 0 \\
& \forall s \in S, h \in H, i \in\left(I_{s} \backslash I_{s}^{\prime}\right), j \in I_{s}(20)
\end{aligned}
$$

Fig. 1 Integrated schedule planning model

seats to be decided by the model and we need to make sure that the total does not exceed the capacity (11) $Q_{k}$ being the capacity of plane type $k$. Constraints (12) maintain that total redirected passengers from itinerary $i$ to all other itineraries including the no-purchase options do not exceed its realized demand. We have already explained the constraints (13) and (14) in the beginning of the section. Constraints (15)-(20) specify the decision variables. Demand value provided by the logit model, $\tilde{d}_{i}^{h}$, serves as an upper bound for the realized demand, $d_{i}^{h}$. The price of each itinerary has an upper bound $U B_{i}$, which is assumed to be the average market price plus the standard deviation. 
A dataset from a major European airline company is used. The model is implemented in AMPL and solved with BONMIN. For large instances computational time becomes unmanageable. For example, for an instance with 78 flights no feasible solution is found in 12 hours. Therefore we propose a Lagrangian relaxation based heuristic in the next section.

\section{Heuristic approach}

We relax the constraints (10) introducing the Lagrangian multipliers $\lambda_{f}^{h}$ for each flight $f$ and class $h$. With this relaxation the model can be decomposed into two subproblems. The first is a revenue maximization model with fare prices modified by the Lagrangian multipliers. The objective function is given by equation (21) subject to constraints (12)-(14) and (18)-(20).

$$
z_{R E V}(\lambda)=\operatorname{Max} \sum_{h \in H} \sum_{f \in F} \sum_{s \in S_{i \in\left(I_{s} \backslash I_{s}^{\prime}\right)}} \delta_{i, f}\left(p_{i}^{h}-\lambda_{f}^{h}\right)\left(d_{i}^{h}-\sum_{\substack{j \in I_{s} \\ i \neq j}} t_{i, j}^{h}+\sum_{\substack{j \in\left(I_{s} \backslash_{s}^{\prime}\right) \\ i \neq j}} t_{j, i}^{h} b_{j, i}^{h}\right)
$$

The second is a fleet assignment model with class-fleet seat prizes. The objective function is given by equation (22) subject to constraints (5)-(9), (11) and (15)-(17).

$$
z_{F A M}(\lambda)=\operatorname{Min} \sum_{k \in K} \sum_{f \in F}\left(C_{k, f} x_{k, f}-\sum_{h \in H} \lambda_{f}^{h} \pi_{k, f}^{h}\right)
$$

The overall procedure is given by algorithm 1 . Upper bound to the problem is obtained by separately solving the problems of $z_{R E V}(\lambda)$ and $z_{F A M}(\lambda)$. Since the capacity constraint is relaxed, we may have infeasibility. Therefore we need to obtain a primal feasible solution which serves as a lower bound. To achieve that we devise a Lagrangian heuristic that uses the optimal solution to $z_{F A M}(\lambda)$ to fix the fleet assignment and class capacity variables. Then it solves a revenue optimization problem with these fixed values dropping the constraints (5)-(9) and (11). This Lagrangian heuristic is included in a neighborhood search loop where neighborhood solutions are explored by changing the fleet assignment of a subset of flights at each iteration. Information provided by the Lagrangian multipliers is used such that flights with high multipliers have higher probability to be selected for the neighborhood search.

The study is in progress and the preliminary results regarding the performance of the heuristic are presented in Table 1. For these small and medium instances, it is seen that we gain computational time and the deviation from the optimal solution is less than $2 \%$. The ongoing work is on the improvement of the heuristic via neighborhood search methods. The heuristic will then be tested for larger data instances. 


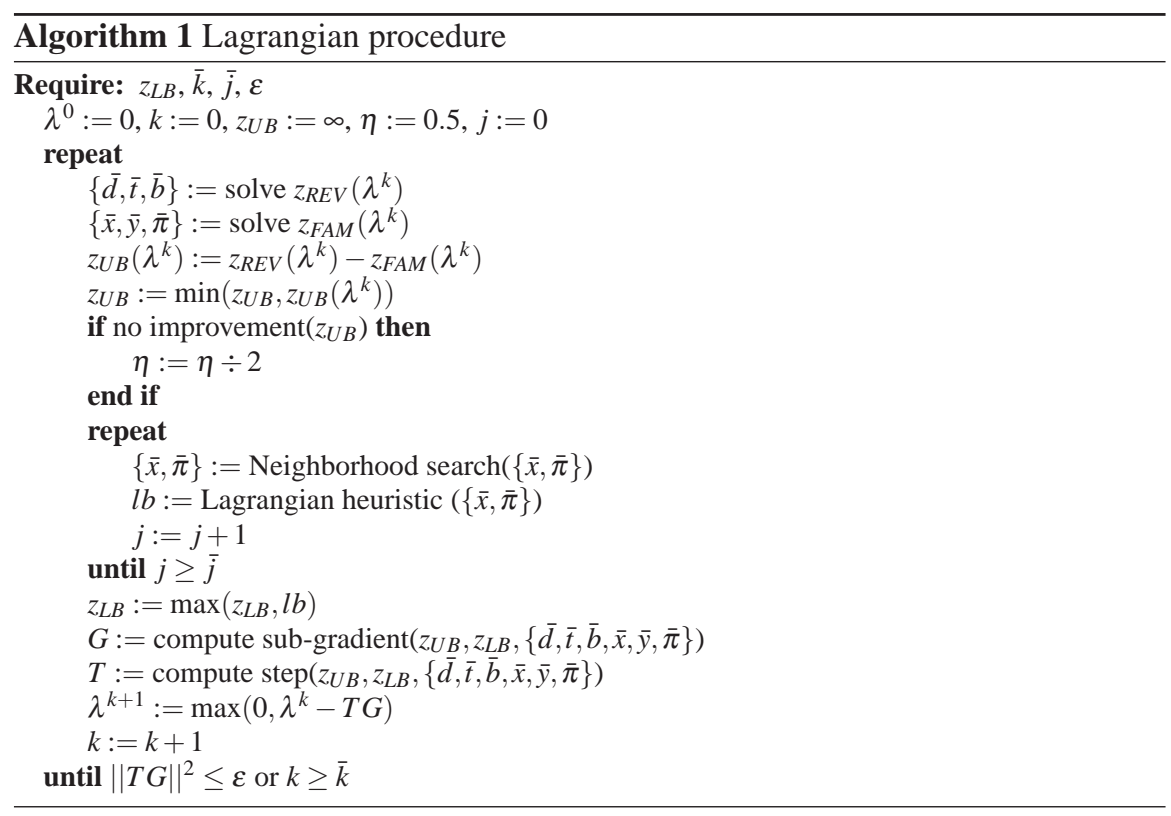

Table 1 Performance of the heuristic

\begin{tabular}{|r|rr|rrr|}
\hline & \multicolumn{2}{|c|}{ BONMIN solver } & \multicolumn{3}{c|}{ Heuristic method } \\
\hline Instances & opt solution time(min) & best solution deviation time(min) \\
\hline 9 flights - 800 pax. & 52,876 & 0.24 & 52,876 & $0 \%$ & 0.07 \\
18 flights - 1096 pax. & 78,275 & 41.04 & 77,224 & $1.34 \%$ & 16.09 \\
26 flights - 2329 pax. & 176,995 & 204.56 & 173,509 & $1.97 \%$ & 41.24 \\
\hline
\end{tabular}

Acknowledgements The authors would like to thank EPFL Middle East for funding the project.

\section{References}

1. Atasoy, B., Salani, M., Leonardi, C., Bierlaire, M.: Clip-Air, a flexible air transportation system. Technical report TRANSP-OR 110929. Transport and Mobility Laboratory, ENAC, EPFL (2011)

2. Barnhart, C., Kniker, T.S., Lohatepanont, M.: Itinerary-based airline fleet assignment. Transportation Science 36, 199-217 (2002)

3. Lohatepanont, M., Barnhart, C.: Airline schedule planning: Integrated models and algorithms for the schedule design and fleet assignment. Transportation Science 38, 19-32 (2004)

4. Schön, C.: Market-oriented airline service design. Operations Research Proceedings, 361$366(2006)$

5. Talluri, K.T., van Ryzin, G.J.: Revenue management under a general discrete choice model of customer behavior. Management Science 50, 15-33 (2004) 